\title{
UFLC-MS/MS analysis of four tanshinone components in Salvia miltiorrhizae after ultrasound-assisted extraction
}

\author{
Shanshan Zhang ${ }^{\mathrm{a}, \mathrm{b}}$, Qiutao Liu ${ }^{\mathrm{a}, \mathrm{b}}$, Hongli Luo ${ }^{\mathrm{c}}$, Ping Chen ${ }^{\mathrm{d}}$, Xiaoru Wu ${ }^{\mathrm{d}}$, \\ Meihua Yang ${ }^{\mathrm{b}}$, Weijun Kong ${ }^{\mathrm{b}, *}$, Weiying Guo ${ }^{\mathrm{a},{ }^{* *}}$
}

${ }^{a}$ Pharmacy College, Liaoning Medical University, Jinzhou 121001, China;

${ }^{\mathrm{b}}$ Institute of Medicinal Plant Development, Chinese Academy of Medical Sciences \& Peking Union Medical College, Beijing, 100193 China;

${ }^{\mathrm{c}}$ Pharmacy College, Chengdu University of Traditional Chinese Medicine, Chengdu 611137, China;

${ }^{\mathrm{d}}$ Guizhou Xinbang Pharmaceutical Co. Ltd., Guiyang 550014,China.

*Correspondence: Prof. W.-J. Kong, E-mail: kongwj302@126.com. Tel.: (86)10 57833277; Fax: (86)10 57833020.

** Correspondence: Prof. W.-Y. Guo, E-mail: wyinguo@163.com. 


\begin{abstract}
A sensitive and rapid ultra-fast liquid chromatography with tandem mass spectrometry (UFLC-MS/MS) method was developed for simultaneous qualitative and quantitative of four characteristic tanshione components including tanshinone IIA, cryptotanshinone, tanshinone I and dihydrotanshinone I in Salvia miltiorrhizae after ultrasound-assisted extraction. By using a $\mathrm{C}_{18}$ column, the four analytes were separated by gradient elution with acetonitrile and water both containing $0.1 \%$ formic acid at the flow rate of $0.3 \mathrm{~mL} / \mathrm{min}$. Multiple-reaction monitoring (MRM) was used for quantification, and an information-dependent acquisition (IDA) method was used to trigger enhanced product ion scans (EPI) for supplementary characteristic identification for qualitative research. Calibration curves showed good linearities with correlation coefficients $(r)$ higher than 0.9990. The method showed high sensitivity with limits of detection (LODs) and quantification (LOQs) less than $0.0002 \mathrm{ng} / \mathrm{mL}$ and $0.0008 \mathrm{ng} / \mathrm{mL}$, respectively, as well as good precision and reproducibility. Mean recoveries for four analytes ranged from $92.5 \%$ to $106.2 \%$ with relative standard deviations (RSDs) lower than $14.59 \%$. Real application of the developed method in 32 batches of $S$. miltiorrhizae samples demonstrated that the total contents of four analytes in all samples were in the range of $2.258-52.342 \mathrm{mg} / \mathrm{g}$. Ultrasound-assisted extraction technique took a small amount of sample and low time but giving high extraction efficiency. Combining with UFLC-MS/MS method in MRM-IDA-EPI mode, more components in other complicated matrices can be simultaneously analyzed for qualitation and quantitation in one run.
\end{abstract}

Keywords: Salvia miltiorrhizae; Tanshinone; Ultrasound-assisted extraction; UFLC-MS/MS; MRM-IDA-EPI 


\section{Introduction}

Salvia miltiorrhizae (Danshen in Chinese and Tanshen in Japanese), is a well-known traditional Chinese medicine (TCM) derived from the dried root of Salvia miltiorrhizae and has been officially recorded in Chinese pharmacopoeia (2010 edition) [1]. It has been widely used in clinic for more than 2000 year in China, Japan, United States and European countries as a common TCM for the treatment of various cardiovascular [2], brain [3], liver [4] and cerebrovascular diseases [5].

In consideration of the important pharmacological activities of $S$. miltiorrhizae, many researchers aimed to study the bioactive components as regard to phenolic compounds such as salvianolic acids [6], flavonoids [7], triterpenoids [8] and lipophilic diterpenoids such as tanshinones [9]. Among them, tanshnones mainly including dihydrotanshinone I, cryptotanshinone and tanshinone I and tanshinone IIA have attracted growing attention due to their strong antibacterial, anti-dermatophytic, anti-neoplastic and anti-platelet aggregation activities [10-11], and the simultaneous determination of tanshinones in S. miltiorrhizae is in great interest.

Currently, different analytical techniques, such as high performance liquid chromatography (HPLC) [12-14], reversed phase liquid chromatography (RP-HPLC) [15], high-speed counter-current chromatography (HSCCC) [16], high-performance thin-layer liquid chromatography (HPTLC) [17], non-aqueous capillary electrophoresis [18], liquid chromatography-tandem mass spectrometry (LC-MS/MS) [19-20], have been developed for the identification and quantitation of various constituents in Salvia miltiorrhizae. Among them, LC-MS/MS with outstanding advantages including low time-consuming and high sensitivity and selectivity has got increasing attention for the multiple components detection in S. miltiorrhizae at very low concentration levels [21], only needing short run time [22] and small consumption of solvents [23]. Recently, a linear ion trap mass spectrometer based LC-QqQ qualitative detection of various analytes in a large crop of complex matrices [24-28], which uses multiple reaction monitoring-information dependent acquisition-enhanced product ion (MRM-IDA-EPI) mode by combining the advantages of QqQ and LIT for multiple targets, trace or ultra-trace chemical residues. MRM-IDA-EPI mode is an innovational target identification method giving higher sensitivity than other modes [29] , as it can provide enhanced sensitivity for each target due to rapid switch between QqQ and LIT. IDA tool can automatically trigger the acquisition of full-scan EPI spectra from the MRM chromatogram of all detected analytes, and MRM 
chromatogram was used as a survey scan for accurate determination and EPI spectra as a dependent scan for supplementary structure identification.

However, prior to analysis of complex S. miltiorrhizae matrix, sample pretreatment is a critical step. As one of the simplest and most inexpensive methods, ultrasound-assisted extraction has been used in many fields, for example wine lees [30], capsicum [31], barbarum [32] and so on because of simple instrument requirements, efficient and environmental protection. It involves the generation of sound waves that trigger extremely high alterations in pressure, leading to the formation of cavitations bubbles, thus damaging the plant matrix and releasing extractive compounds [33]. Being attributed to the effect of acoustic cavitations produced in the solvent by the passage of the ultrasound wave, the extraction efficacy of targets from complex matrices is enhanced [34].

In the present study, a sensitive and selective ultrasound-assisted extraction procedure based UFLC-ESI-QqQ ${ }_{\text {LIT }}$-MS/MS method equipped with a turbo ion spray interface in positive ionization mode $\left(\mathrm{ESI}^{+}\right)$was developed and this method successfully applied to qualitative and quantification of four tamshinone components (dihydrotanshinone I, cryptotanshinone and tanshinone I and tanshinone IIA) in 32 batches of $S$. miltiorrhizae samples including top-geoherbs and raw material samples collected from different cities of China. By employing an IDA approach through MRM chromatogram as survey scan and EPI spectra as dependent scan for characteristic identification, the target analytes have got simultaneously qualitative and quantitative analysis in one run, which provided a valuable method and idea reference for quality evaluation of TCMs.

\section{Experimental}

\subsection{Chemicals and materials}

Methanol and acetonitrile (HPLC grade) were obtained from Fisher Scientific (Fair Lawn, NJ, USA). Formic acid was bought from Xilong Chemical (Guangdong, China). Purified water was used (Wahaha, Hangzhou, China). A KQ-500 ultra-sonic cleaning bath $(50 \times 30 \times 35 \mathrm{~cm})$ was manufactured by Kunshan Ultrasonic Instrument Co., Ltd. (Jiangsu, China).

The four standards of dihydrotanshinone I, cryptotanshinone and tanshinone I were provided by Chengdu Must Bio-Technology, Chengdu, China. Tanshinone IIA was purchased from National Institutes for Food and Drug Control, Beijing, China. Their purities were all above $98 \%$.

Six top-geoherbs of S. miltiorrhizae (S1-S6) and the rest of 3 batches (S7-S9), 4 batches (S10-S13), S14 and S15 were purchased from Zhongjiang city, Sichuan province, Henan province, 
Shandong province, and Tongrentang and Hongshengtang in Beijing province, respectively. And the definitive 17 batches of raw material samples of S. miltiorrhizae. (S16-S32) were offered by Guizhou Xinbang Pharmaceutical Co. Ltd., which were authenticated by Prof. Yulin Lin (Institute of Medicinal Plant Development, Chinese Academy of Medical Sciences and Peking Union Medical College, China). All samples were collected in sterilized polyethylene bags and stored at $-20{ }^{\circ} \mathrm{C}$ before analysis.

\subsection{Standard preparation}

The mixed stock solution was prepared by dissolving each standard in methanol to obtain 200 $\mathrm{ng} / \mathrm{mL}$ of dihydrotanshinone I, $500 \mathrm{ng} / \mathrm{mL}$ of cryptotanshinone, $500 \mathrm{ng} / \mathrm{mL}$ of tanshinone I and 200 $\mathrm{ng} / \mathrm{mL}$ of tanshinone IIA, and the working standard solutions were freshly prepared by diluting suitable amounts of the above-prepared stock solution with methanol. All solutions were stored at 4 ${ }^{\circ} \mathrm{C}$ in a refrigerator.

\subsection{Sample preparation}

An aliquot of $0.2 \mathrm{~g}$ dried $S$. miltiorrhizae powder (through a 50-mesh sieve) was accurately weighed and transferred into a 100-mL flask. After being added with $50 \mathrm{~mL}$ of methanol, the flask was weighed and changed into the ultra-sonic cleaning bath for extraction for $30 \mathrm{~min}$ at room temperature. Then, the lost weight was made up and the mixture was centrifuged at $4000 \mathrm{rpm}$ for 10 min. afterward, $50 \mu \mathrm{L}$ of the supernatant was transferred into a $2.5-\mathrm{mL}$ centrifuge tube for dilution with $1 \mathrm{~mL}$ of methanol. The above procedure was repeated. Finally, the extract was filtered through a $0.22 \mu \mathrm{m}$ filter membrane for UFLC-MS/MS analysis. All solutions were stored at $4{ }^{\circ} \mathrm{C}$ in the dark until analysis.

\subsection{Instrumentation}

An ultra-fast liquid chromatography (UFLC) system including two LC-20ADXR pumps, a DGxU-20 A3 degasser, a SIL-20AC auto-sampler and a CTO-20A column oven from Shiseido Technologies (Shimadzu, Kyoto, Japan) was used. The analytes were separated on a SHISEIO CAPCELL CORE $\mathrm{C}_{18}$ column $(50 \times 2.1 \mathrm{~mm}$ I.D, $2.7 \mu \mathrm{m}$; Shiseio, Japan). The mixture of (A) $0.1 \%$ formic acid in acetonitrile and (B) $0.1 \%$ formic acid in water was chosen as the mobile phase using a gradient program: initial $90 \% \mathrm{~B} ; 3 \mathrm{~min}, 70 \% \mathrm{~B} ; 8-12 \mathrm{~min}, 10 \% \mathrm{~B} ; 12.01 \mathrm{~min}, 90 \% \mathrm{~B}$ and stayed at $90 \% \mathrm{~B}$ for $3 \mathrm{~min}$. The flow rate was $0.3 \mathrm{~mL} / \mathrm{min}$ and the injection volume was $1.0 \mu \mathrm{L}$.

An Applied Biosystems Sciex 5500 QTRAP ${ }^{\circledR}$ mass spectrometer system (Foster City, CA, USA) 
equipped with an electrospray ionization (ESI) source was coupled to the UFLC system described above. The MS/MS detection was operated in positive ESI modes. Nitrogen was used as the nebulizer (GS1), heater (GS2) and curtain (CUR) gases and the collision activation dissociation (CAD) gas. Tandem MS analyses were performed in the MRM acquisition mode with two precursor-to-product ion transitions monitored for simultaneous detection of all the analytes. The MS/MS parameters were optimized and finally set as follows: source temperature: $550{ }^{\circ} \mathrm{C}$, ion spray (IS) voltage: +5500 V, GS1: 50psi, GS2: 50 psi, CUR: 35 psi, CAD: medium.

An IDA experiment was employed to automatically trigger EPI scans by analyzing MRM signals. The EPI scans were operated in the $\mathrm{ESI}^{+}$mode for product ions at a scan rate $10000 \mathrm{Da} / \mathrm{s}$ with dynamic fill in the linear ion trap and a step size of $0.12 \mathrm{Da}$. The collision energy spread (CEs) of EPI was set at two continues energy levels of $15 \mathrm{eV}$ to provide rich EPI spectra. For the MRM-EPI analysis, the IDA criteria were set to allow the two most intense product ions of each analyte that exceeded 1000 counts per seconds (cps) with dynamic exclusion of the former ions for 15s. The Applied Biosystems Analyst software (version 1.6) was used to control the UFLC-MS/MS system and acquire the data.

\subsection{Method validation}

To evaluate the method suitability, the established UFLC-MS/MS was validated in terms of specificity, selectivity, calibration curves, limit of detection (LOD), limit of quantification (LOQ), precision, recovery, stability and reproducibility according to the guidelines of the U.S. FDA, International Conference on Harmonization (ICH) and some reports [35-38]. Sample 7 (S7) was randomly selected for this validation.

\subsubsection{Specificity and selectivity}

To verify the absence of interference substances, the MRM chromatograms of the blank solvent, solution of mixed standards and sample solution were compared regarding to their retention time with the target analytes. Typical LC-MS/MS MRM chromatograms in Fig. 1 demonstrated that the four analytes have got good separation and excellent repeatability with no interference peaks.

Fig. 1.

\subsubsection{Calibration curve, linearity, $L O D$ and $L O Q$}

The mixed stock solution was diluted to obtain a sequence of solutions $(0.0008,0.004,0.008$, $0.02,0.04,0.08,0.2,0.4,0.8,1.6,4,8,20,40,80,200 \mathrm{ng} / \mathrm{mL}$ of dihydrotanshinone I and tanshinone 
IIA; $0.002,0.01,0.02,0.05,0.1,0.2,0.5,1,2,4,10,20,50,100,200,500 \mathrm{ng} / \mathrm{mL}$ of cryptotansinone and tanshinone I) for UFLC-MS/MS analysis. Calibration curves were established using the peak areas as the dependent variable ( $y$-axis) and concentration of each analyte as the independent variable ( $x$-axis). The linearity was evaluated by the correlation coefficient ( $r$ ) value of each calibration curve. The LOD and LOQ for each analyte were calculated at signal-to-noise $(\mathrm{S} / \mathrm{N})$ of 3 and 10 , respectively, by analyzing several decreasing concentrations of each analyte until the $\mathrm{S} / \mathrm{N}$ was reached.

\subsubsection{Precision}

Intra- and inter-day variations were determined for the precision of the established method. Intra-day precision was evaluated by six successive injection of S7 solution on the same day, while inter-day precision was determined on five consecutive days, and the variation was expressed as relative standard deviation $(\mathrm{RSD})$.

\subsubsection{Accuracy}

Recovery experiments were performed to evaluate the method accuracy. The four standards were added to S7 sample at three different concentration levels (approximately equivalent to $0.8,1.0$ and 1.2 times of the concentration of them in the matrix) to examine recovery for each analyte and each level was analyzed in triplicate. The fortified samples were extracted and analyzed according to the above procedure. The average recovery was estimated by the following equation:

$$
\text { Recovery }(\%)=\frac{\text { amount found }- \text { original amount }}{\text { amount spiked }} \times 100 \%
$$

\subsubsection{Stability and reproducibility}

The stability of $\mathrm{S} 7$ solution stored at $4{ }^{\circ} \mathrm{C}$ was evaluated at the time points of $0,2,4,8,24$ and $48 \mathrm{~h}$. The experimental concentrations were compared with the normal values got the corresponding contents. The reproducibility of the quantitative procedure was based on the results of six portions of one batch of S7 sample. The variation is expressed as RSD of stability and reproducibility of four diterpenoids.

\section{Results and discussion}

\subsection{Method development}

Based on the experimental analysis, more attention should be paid to the method development including the optimization of extraction procedures and UFLC-MS/MS conditions. 


\subsubsection{Optimization of extraction procedure}

In order to establish a suitable method for extraction of tanshinone components from $S$. miltiorrhizae, different extraction solvents (methanol, acetonitrile and different ratios of them with water), extraction modes (ultrasonic and reflux), together with extraction time (30 min, $45 \mathrm{~min}$ and $60 \mathrm{~min}$ ) were investigated. It was found that good recovery and rapid extraction can be obtained by ultrasonic with methanol. Afterward, the influence of extraction time (30 min, $45 \mathrm{~min}$ and $60 \mathrm{~min}$ ) and cycle (one or two times) on the recovery of all analytes were experimentally tested. The results showed that there were no significant differences among these conditions. Thus, considering the time and cost saving, this study select the conditions that were extracted based on methanol and the ultrasonic as presented in Section 2.3.

\subsubsection{Optimization of UFLC-MS/MS conditions}

In this study, both negative and positive ESI modes have been used to determine the response of target analytes with high sensitivity. The results demonstrated that the analytes in $\mathrm{ESI}^{+}$mode expressing high intensity and good sensitivity of precursor and product ions. Then, the MS/MS parameters including declustering potential (DP), collision energy (CE), and collision cell exit potential (CXP) for each analyte, as well as their retention time has been optimized and described in Table 1.

Table 1

Afterward, the UFLC conditions including the type of chromatographic column and the composition of mobile phase were optimized. The results have confirmed that the mobile phase of $0.1 \%$ formic acid in water/acetonitrile flowing through the SHISEIO CAPCELL CORE $\mathrm{C}_{18}$ chromatographic column $(50 \times 2.1 \mathrm{~mm}$ I.D, $2.7 \mu \mathrm{m})$ could provide good separation and high MS/MS response of the four analytes. The gradient elution condition was also optimized and listed in the above section.

\section{2. $\quad$ Method validation}

\subsubsection{Specificity and selectivity}

According to the UFLC-MS/MS MRM chromatograms, it could be concluded that there were no interference peaks from other constituents at the retention time of the four analytes $(2.70 \mathrm{~min}$, $3.03 \mathrm{~min}, 3.10 \mathrm{~min}$ and $3.39 \mathrm{~min}$ for dihydrotanshinone I, cryptotanshinone, tanshinone I and tanshinone IIA, respectively). In addition, the responses of the four analytes in the MRM 
chromatograms were enough high for quantitation, demonstrating high specificity and good sensitivity of the method.

\subsubsection{Calibration curve, $L O D$ and $L O Q$}

Four non-zero calibration curves were constructed in the concentration ranges of $0.02-20 \mathrm{ng} / \mathrm{mL}$ for dihydrotanshinone I, cryptotanshinone and tanshinone I, and 0.0008-8 ng/mL for tanshinone IIA. Within the define calibration ranges, satisfactory linearity of each calibration curve was observed for all the four analytes with $r$ greater than 0.9990 . Also, the LOD and LOQ ranged in 0.0002-0.004 and 0.0008-0.02, respectively. The results were shown in Table 2.

Table 2

\subsubsection{Precision}

The RSDs of peak areas, which were taken to evaluate the intra- and inter-day precision values for the method, were less than $8.82 \%$ (Table 2) for all analytes, indicating that the UFLC-MS/MS method was precise and sensitive for the quantitative analysis.

\subsubsection{Recovery}

All the analytes showed high recovery $(92.5 \%-106.2 \%)$ in the fortified samples at three spiking levels with RSDs less than $14.59 \%$. Data summarized in Table 3 have indicated that the measurement for the four analytes is accurate.

\section{Table 3}

\subsubsection{Stability and reproducibility}

The results in Table 2 have proved satisfactory stability of the sample solution at $4{ }^{\circ} \mathrm{C}$. Reproducibility ( $n=6)$ samples were all run and the RSD (\%) values were calculated as seen in Table 2. Satisfactory results were obtained with RSD of peak areas lower than $14.05 \%$, indicating good reproducibility of the chromatographic separation.

\subsection{Confirmation of target analytes}

With the help of IDA experiment, the UFLC-MS/MS MRM chromatogram of S. miltiorrhizae sample regarding the four tanshinone components was triggered to obtain synchronous EPI spectra, which could be used to confirm the targets. From Fig. 2, it could be seen that tanshinone IIA, cryptotanshinone, tanshinone I and dihydrotanshinone I at 2.70, 3.03, 3.10 and $3.39 \mathrm{~min}$ expressed obvious protonated molecular ion of $\mathrm{m} / \mathrm{z} 261.2,254.1,249$ and 277.3, respectively. The corresponding product ions for each analyte were in line with that listed in Table 1. Then, the target 
analytes could get simultaneously qualitative and quantitative analysis in one run to ensure the accuracy and reliability of final data.

Fig. 2.

\subsection{Analysis of real samples}

The developed UFLC-ESI ${ }^{+}$-MS/MS method based on MRM-IDA-EPI mode was successfully applied for the simultaneous determination of four tanshinone components in 32 batches of $S$. miltiorrhizae samples. Therefore, compared the content of constituents to provide the basis for the evaluation and identification of quality of raw-material samples are provided by four different provinces. Form Table 4, it could be seen that the content of the each and total of components in samples, and showed that the 32 batches of S. miltiorrhiza Bunge samples experimental results conform to the rules in Table 4. Of them, dihydrotanshinone I, cryptotanshinone, tanshinone I and tanshinone IIA are present in the highest amount [39], while their contents in different samples showed significant difference. As can be seen from the Table 4 shows, the total contents of 17 bottom-geoherbs of S. miltiorrhiza samples (S16-S32) are generally higher than others which more than 12.29. However, although the total content of four components in S15 and S16 were 2.258 $\mathrm{mg} / \mathrm{g}$ and $2.636 \mathrm{mg} / \mathrm{g}$ much lower than others in 32 batches of samples, the content of dihydrotanshinone I and tanshinone I were not the lowest. In addition, the contents of same origin of samples also have a great difference, for example S7-S9, the total contents were 6.318, 2.774 and $4.767 \mathrm{mg} / \mathrm{g}$, respectively. All the contents were calculated with standard method, and the total values from four components were summarized in Table 4. It show data from different samples collected from different cities during our study. The variation of chemical compositions might be attributed to the different geographic and environmental conditions, further lead to the quality differences of the test $S$. miltiorrhizae samples.

Table 4

\section{Conclusion}

In conclusion, an efficient and convenient UFLC-MS/MS method has been developed for the simultaneous determination of dihydrotanshinone I, cryptotanshinone, tanshinone I, tanshinone IIA in S. miltiorrhizae. With MRM-IDA-EPI mode, qualitative and quantitative analysis of target compounds was much clear showed results. Therefore, this method, with both convenience and analysis time were taken into consideration in the optimization procedure, uses the optimized 
methodology based on the dilution of methanol as extraction solution and most importantly that is simple, efficient, cheaper and less time consuming. The low enough of LODs and LOQs for quantification of five compounds in S. miltiorrhizae were further confirmed the effective and efficient method. The present study gathered the data to help identify the content of major lipophilic constituents for S. miltiorrhizae and provide important information for the following pharmacological experiments and pharmaceutical research which will be helpful in better understanding the effective action of S. miltiorrhizae.

\section{Acknowledgements}

This study was supported by the National Science Foundation of China $(81274072,81473346)$ and Xiehe New Star Project.

\section{References}

[1] Y.B. Guo, Y.L, L.M. Xue, R.P. Severino, S.H. Gao, J.Z. Niu, L.P. Qin, D.W. Zhang, D. Brömme, J. Ethnopharmacol. 155 (2014 ) 1401-1416.

[2] T.O. Cheng, Int. J. Cardiol. 121 (2007) 9-22.

[3] M.F. Zeng, L.M. Pan, S.M. Qi, Y.T. Cao, H.X. Zhu, L.W. Guo, J. Zhou, Fitoterapia 88 (2013) $50-75$.

[4] C.F. Chien, Y.T. Wu, T.H. Tsai, Biomed. Chromatogr. 25 (2011) 21-38.

[5] X.P. Chen, J.J. Guo, J.L. Bao, J.J. Lu, Y.T. Wang, Med. Res. Rev. 34 (2014) 768-794(27).

[6] L.M. Zhou, Z. Zuo, M.S.S. Chow, J. Clin. Pharmacol. 45 (2005) 1345-1359.

[7] X.B. Wang, S.L. Morris-Natscbke, K.H. Lee, Med. Res. Rev. 27 (2007) 133-148.

[8] Y.G. Li, L. Song, M. Liu, Z.B. Hu, Z.T. Wang, J. Chromatogr A 1216 (2009) 1941-1953.

[9] Y.R. Lu, L.Y. Foo, Phytochemistry 59 (2002) 117-140.

[10] L. Cui, T. Wu, Y.Y. Liu, Y.F. Deng, C.M. Ai, H.Q. Chen, Acta Pharmacol. Sin. 25 (2004) $678-684$.

[11] S.L. Yuan, Y.Q. Wei, X.J. Wang, F. Xiao, S.F. Li, J. Zhang, World J. Gastroenterol. 10 (2004) 2024-2028.

[12] A.H. Liu, Y.H. Lin, M. Yang, J.H. Sun, H. Guo, D.A. Guo, J. Pharmaceut. Sci. 9 (2006) 1-9.

[13] M. Liu, Y.G. Li, G.X. Chou, X.M. Cheng, M. Zhang, Z.T. Wang, J. Chromatogr A 1157 (2007) 51-55.

[14] S.Q. Gao, W. Yu, X. Yang, Z.L. Liu, Y.L. Jia, H.Q. Zhang, J. Sep. Sci. 35 (2012) 2813-2821. 
[15] Y.J. Yan, G.D. Yang, L.C. He, Chin. Tradit. Herb. Drugs 33 (2002) 363-365.

[16] M. Gu, G.F. Zhang, Z.G. Su, F. Ouyang, J. Chromatogr A 1041 (2004) 239-243.

[17] J. Yang, L.L. Choi, D.Q. Li, F.Q. Yang, L.J. Zeng, J. Zhao, S.P. Li, Jpc-J. Planar. Chromat. 24 (2011) $257-263$.

[18] M. Gu, S.F. Zhang, Z.G. Su, Y. Chen, F. Ouyang, J. Chromatogr A 1057 (2004) 133-140.

[19] J. Cao, Y.J. Wei, L.W. Qi, P. Li, Z.M. Qian, H.W. Luo, J. Chen, J. Zhao, Biomed. Chromatogr. 22 (2008) 164-172.

[20] P. Hu, G.A. Luo, Z.Z. Zhao, Z.H. Jiang, Chem. Pharm. Bull. 53 (2005) 705-709.

[21] O. Núñez, E. Moyano, M.T. Galceran, TrAC-Trends. Anal. Chem.24 (2005) 683-703.

[22] N. Koseki, H. Kawashita, M. Niina, Y. Nagae, N. Masuda, J. Pharmaceut. Biomed. 36 (2005) 1063-1072.

[23] J. Bhatt, S. Singh, G. Subbaiah, B. Shah, S. Kambli, S. Ameta, Biomed. Chromatogr. 21 (2007) 169-175.

[24] W.J. Kong, J. Wen, Y.H. Yang, F. Qiu, P. Sheng, M.H. Yang, Analyst 139 (2014) 1883.

[25] Z.W. Zhu, J. Li, X.M. Gao, E. Amponsem, L.M. Hu, B.L. Zhang, Y.X. Chang, J. Pharmaceut. Biomed. 62 (2012) 162-166.

[26] J.S. Lee, D.H. Kim, K.H. Liu, T.K. Oh, C.H. Lee, Rapid Commun. Mass Sp. 19 (2005) 3539-3548.

[27] T.H. Kao, S.C. Huang, B.S. Inbaraj, B.H. Chen, Anal. Chim. Acta 626 (2008) 200-211.

[28] P. Montoro, C.I.G. Tuberoso, M. Maldini, P. Cabras, C. Pizza, Nat. Prod. Commun. 3 (2008) 2013-2016.

[29] M. Yao, H.J. Zhang, S. Chong, R.A. Morrison, J. Pharmaceut. Biomed. 32 (2003) 1189-1197.

[30] E. Naziri, F. Mantzouridou, M.Z. Tsimidou, J. Agr. Food Chem. 60 (2012) 9195-9201.

[31] S. Boonkird, C. Phisalaphong, M. Phisalaphong, Ultrasonics Sonochem. 15 (2008) 1075-1079.

[32] G. Zhang, G.Q. Zhao, Food Sci. 9 (2005) 073.

[33] D. Horžić, A.R. Jambrak, A. Belščak-Cvitanović, D. Komes, V. Lelas, Food Bioprocess Tech. 5 (2012) 2858-2870.

[34] J. Wang, B.G. Sun, Y.P. Cao, Y. Tian, X.H. Li, Food Chem. 106 (2008) 804-810.

[35] International conference on harmonization (ICH). Q2 (R1): Text on validation of analytical procedures, 2005, 
[36] US Food and Drug Administration, Analytical Procedures and Methods Validation, 2000,

[37] X.X. Diao, Z.Y. Ma, P. Lei, D.F. Zhong, Y.F. Zhang, X.Y. Chen, J. Chromatogr B 939(2013) $67-72$.

[38] X.X. Diao, Z.Y. Ma, H.D. Wang, D.F. Zhong, Y.F. Zhang, J. Jin, Y.X. Fan, X.Y. Chen, J. Pharmaceut. Biomed. 78(2013) 19-26.

[39] Z.H. Shi, J.T. He, T.T. Yao, W.B. Chang, M.P. Zhao, J. Pharmaceut. Biomed. 37 (2005) 481-486. 
Figure legends

Fig. 1. Typical LC-MS/MS MRM chromatograms of (A) standard solution of each analyte (200 $\mathrm{ng} / \mathrm{mL}$ of dihydrotanshinone I and tanshinone IIA, $500 \mathrm{ng} / \mathrm{mL}$ of tanshinone I and cryptotanshinone), (B) blank solution, and (C) S. miltiorrhizae sample.

Fig. 2. EPI spectra for synchronous confirmation of the four target analytes in S. miltiorrhizae based on MRM-IDA-EPI mode. 

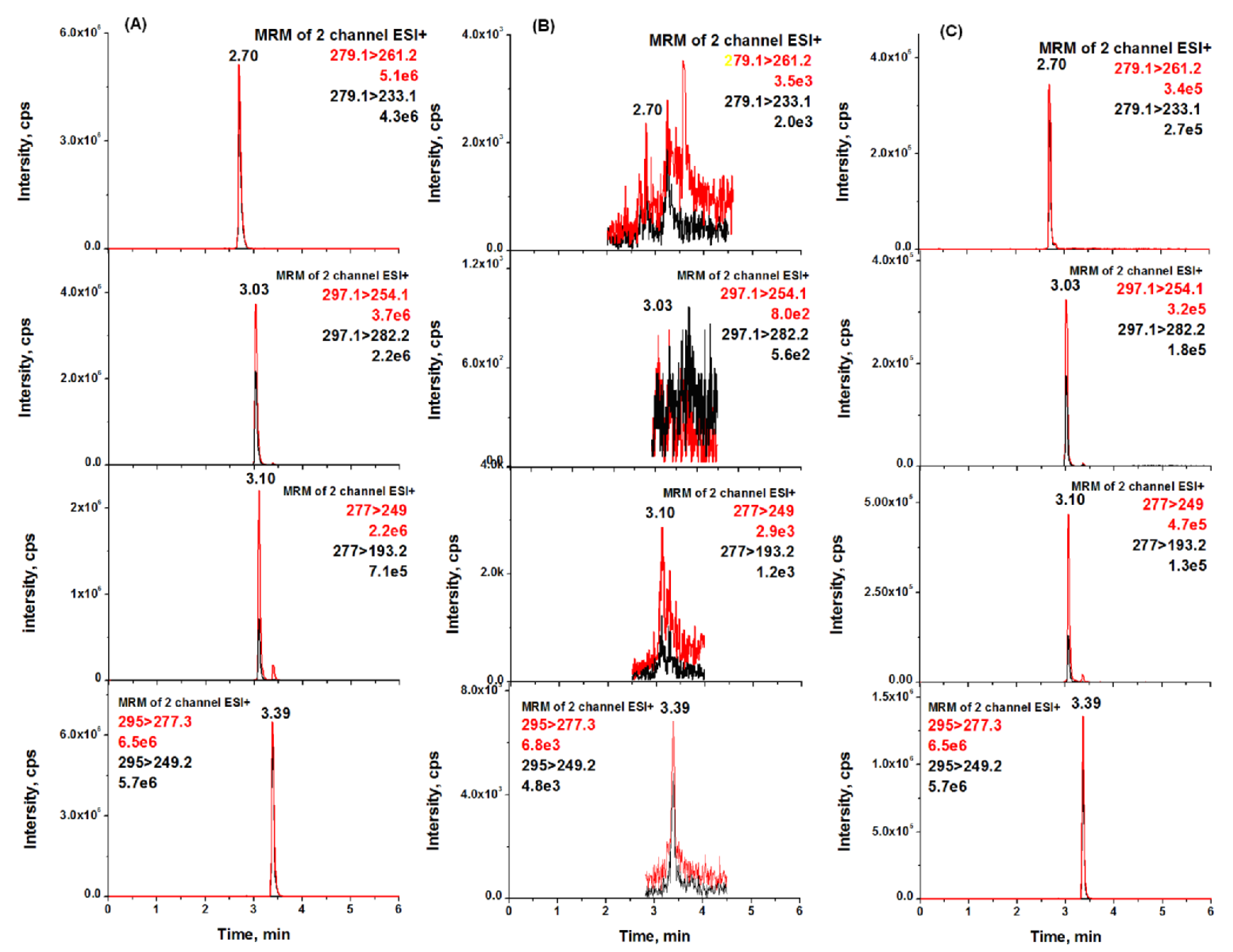

Fig. 1 

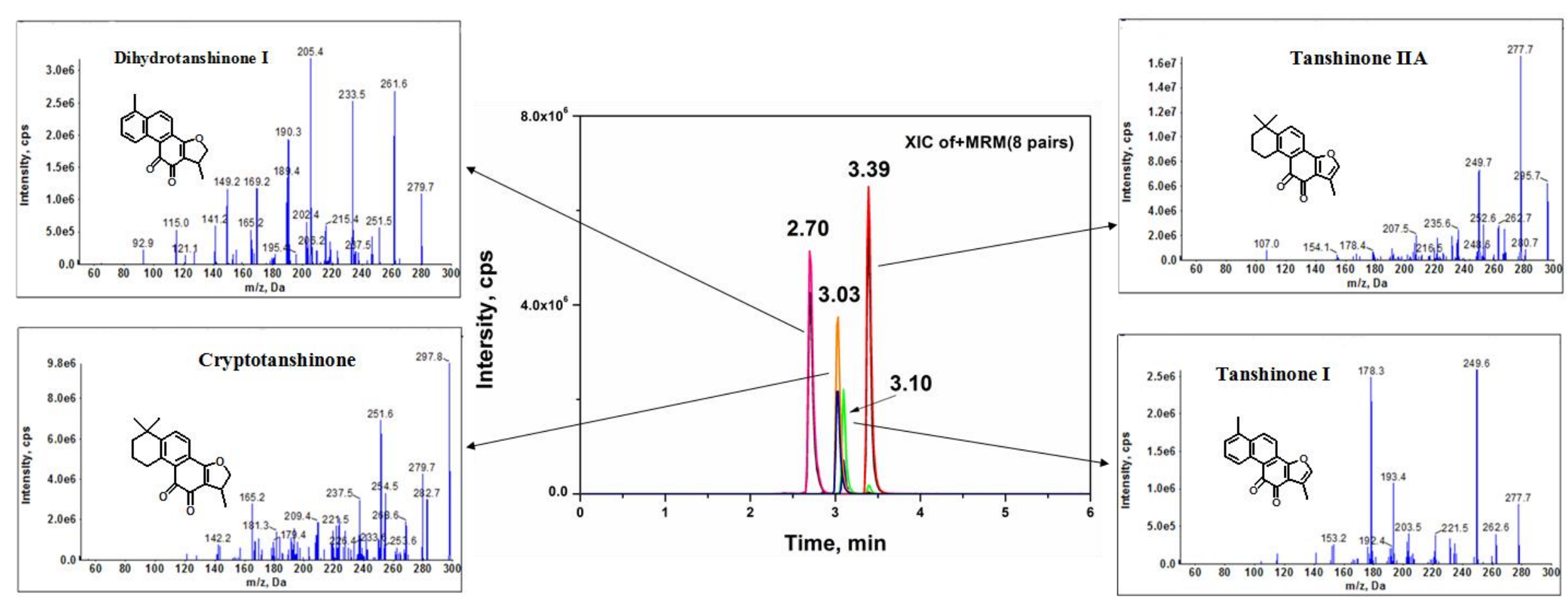

Fig. 2 
Table 1. MS/MS parameters of test analytes

\begin{tabular}{|c|c|c|c|c|c|c|c|}
\hline Analytes & $\mathrm{RT}^{\mathrm{a}}(\min )$ & Precursor ions & Product ions & $\mathrm{DP}^{\mathrm{b}}(\mathrm{V})$ & $\mathrm{CE}^{\mathrm{c}}(\mathrm{V})$ & $\mathrm{CXP}^{\mathrm{d}}(\mathrm{V})$ & Dwell time (ms) \\
\hline \multirow{2}{*}{ Dihydrotanshinone I } & \multirow{2}{*}{2.70} & \multirow{2}{*}{279.1} & $261.2^{*}$ & \multirow{2}{*}{160} & 25 & 11 & 80 \\
\hline & & & 233.1 & & 30 & 11 & 80 \\
\hline \multirow{2}{*}{ Cryptotanshinone } & \multirow{2}{*}{3.03} & \multirow{2}{*}{297.1} & $254.1^{*}$ & \multirow{2}{*}{160} & 37 & 7 & 80 \\
\hline & & & 282.2 & & 38 & 7 & 80 \\
\hline \multirow{2}{*}{ Tanshinone I } & \multirow{2}{*}{3.10} & \multirow{2}{*}{277} & $249^{*}$ & \multirow{2}{*}{160} & 27 & 13 & 80 \\
\hline & & & 193.2 & & 35 & 13 & 80 \\
\hline \multirow{2}{*}{ Tanshinone IIA } & \multirow{2}{*}{3.39} & \multirow{2}{*}{295} & $277.3^{*}$ & \multirow{2}{*}{110} & 27 & 7 & 80 \\
\hline & & & 249.2 & & 31 & 7 & 80 \\
\hline
\end{tabular}

${ }^{\mathrm{a}} \mathrm{RT}$ : retention time; ${ }^{\mathrm{b}} \mathrm{DP}$ : declustering potential; ${ }^{\mathrm{c}} \mathrm{CE}$ : collision energy; ${ }^{\mathrm{d}} \mathrm{CXP}$ : cell exit potential; ${ }^{\mathrm{P}}$ Product ions. 
Table 2. LC-MS/MS method validation for test analytes.

\begin{tabular}{|c|c|c|c|c|c|c|c|c|}
\hline \multirow{2}{*}{ Analytes } & \multirow{2}{*}{ Calibration curves } & \multirow{2}{*}{$\begin{array}{c}\text { Linear range } \\
\qquad(\mathrm{ng} / \mathrm{mL})\end{array}$} & \multirow{2}{*}{$r$} & \multirow{2}{*}{$\begin{array}{c}\text { LOD } \\
(\mathrm{ng} / \mathrm{mL})\end{array}$} & \multirow{2}{*}{$\begin{array}{c}\text { LOQ } \\
(\mathrm{ng} / \mathrm{mL})\end{array}$} & \multicolumn{3}{|c|}{ RSD (\%) } \\
\hline & & & & & & Stability & Reproducibility & Precision \\
\hline Dihydrotanshinone I & $y=66500 x+24800$ & $0.02-20$ & 0.9991 & 0.004 & 0.02 & 4.69 & 3.34 & 4.16 \\
\hline Cryptotanshinone & $y=126000 x+23900$ & $0.02-20$ & 0.9998 & 0.002 & 0.02 & 13.76 & 14.05 & 8.82 \\
\hline Tanshinone I & $y=136000 x+64200$ & $0.02-20$ & 0.9993 & 0.002 & 0.02 & 6.54 & 2.61 & 3.58 \\
\hline Tanshinone IIA & $y=153000 x+98500$ & $0.0008-8$ & 0.9990 & 0.0002 & 0.0008 & 5.44 & 3.14 & 7.58 \\
\hline
\end{tabular}


Table 3. Recovery data by LC-MS/MS method $(n=3)$.

\begin{tabular}{|c|c|c|c|c|c|}
\hline Analytes & Original $(\mu \mathrm{g})$ & Spiked $(\mu \mathrm{g})$ & Found $(\mu \mathrm{g})$ & Mean recovery $(\%)$ & $\operatorname{RSD} \%(\mathrm{n}=3)$ \\
\hline & & 54.272 & 53.295 & 98.2 & 6.61 \\
\hline \multirow[t]{3}{*}{ Dihydrotanshinone I } & 67.84 & 67.84 & 64.991 & 95.8 & 3.34 \\
\hline & & 81.408 & 79.454 & 97.6 & 4.51 \\
\hline & & 61.953 & 64.617 & 104.3 & 11.62 \\
\hline \multirow[t]{3}{*}{ Cryptotanshinone } & 77.468 & 77.468 & 82.271 & 106.2 & 14.05 \\
\hline & & 92.983 & 92.053 & 99.0 & 14.59 \\
\hline & & 199.28 & 194.896 & 97.8 & 7.46 \\
\hline \multirow[t]{3}{*}{ Tanshinone I } & 249.1 & 249.1 & 230.418 & 92.5 & 2.61 \\
\hline & & 298.92 & 283.077 & 94.7 & 2.73 \\
\hline & & 124.46 & 127.198 & 102.2 & 5.01 \\
\hline \multirow[t]{2}{*}{ Tanshinone IIA } & 155.82 & 155.82 & 161.897 & 103.9 & 3.14 \\
\hline & & 186.2 & 180.242 & 96.8 & 2.89 \\
\hline
\end{tabular}


Table 4. Contents of four analytes in 13 batches of samples $(\mathrm{mg} / \mathrm{g})$.

\begin{tabular}{|c|c|c|c|c|c|}
\hline No. & Dihydrotanshinone I & Cryptotanshinone & Tanshinone I & Tanshinone IIA & Total \\
\hline $\mathrm{S} 1$ & 0.491 & 0.411 & 0.468 & 3.296 & 4.666 \\
\hline S2 & 0.160 & 0.212 & 0.288 & 2.598 & 3.257 \\
\hline S3 & 0.140 & 0.192 & 0.266 & 3.007 & 3.604 \\
\hline $\mathrm{S} 4$ & 0.255 & 0.369 & 0.337 & 4.308 & 5.269 \\
\hline S5 & 0.179 & 0.289 & 0.265 & 3.497 & 4.230 \\
\hline S6 & 0.325 & 0.398 & 0.308 & 3.315 & 4.345 \\
\hline S7 & 1.216 & 0.771 & 0.872 & 3.459 & 6.318 \\
\hline S8 & 0.754 & 0.359 & 0.258 & 1.403 & 2.774 \\
\hline S9 & 0.935 & 0.653 & 0.575 & 2.604 & 4.767 \\
\hline S10 & 0.295 & 0.518 & 0.319 & 2.541 & 3.672 \\
\hline S11 & 1.129 & 1.634 & 0.838 & 4.478 & 8.080 \\
\hline $\mathrm{S} 12$ & 0.963 & 1.736 & 0.705 & 4.610 & 8.015 \\
\hline S13 & 0.528 & 1.267 & 0.694 & 4.730 & 7.219 \\
\hline S14 & 0.345 & 0.159 & 0.301 & 1.453 & 2.258 \\
\hline S15 & 0.298 & 0.257 & 0.383 & 1.698 & 2.636 \\
\hline S16 & 3.860 & 3.487 & 2.205 & 14.346 & 23.898 \\
\hline S17 & 2.231 & 2.484 & 1.746 & 12.333 & 18.795 \\
\hline S18 & 1.813 & 1.427 & 1.190 & 8.321 & 12.752 \\
\hline S19 & 1.188 & 1.050 & 1.168 & 9.491 & 12.897 \\
\hline S20 & 1.395 & 1.492 & 1.057 & 8.346 & 12.290 \\
\hline S21 & 1.741 & 1.988 & 1.161 & 11.340 & 16.230 \\
\hline S22 & 1.813 & 2.560 & 1.435 & 10.119 & 15.927 \\
\hline S23 & 2.217 & 1.697 & 1.368 & 9.151 & 14.433 \\
\hline S24 & 2.433 & 2.333 & 1.368 & 10.057 & 16.191 \\
\hline S25 & 8.156 & 4.835 & 5.887 & 33.465 & 52.342 \\
\hline S26 & 4.033 & 4.921 & 2.924 & 21.893 & 33.771 \\
\hline S27 & 2.347 & 2.635 & 2.205 & 16.358 & 23.545 \\
\hline S28 & 1.655 & 3.228 & 2.694 & 21.264 & 28.841 \\
\hline S29 & 2.361 & 3.799 & 2.146 & 14.094 & 22.401 \\
\hline S30 & 1.323 & 2.074 & 2.257 & 17.239 & 22.894 \\
\hline S31 & 2.592 & 2.958 & 1.339 & 11.566 & 18.455 \\
\hline S32 & 3.183 & 3.239 & 2.613 & 16.862 & 25.896 \\
\hline
\end{tabular}

